\title{
Frustration-induced supersolids in the absence of intersite interactions
}

\author{
Tapan Mishra, Sebastian Greschner, and Luis Santos \\ Institut für Theoretische Physik, Leibniz Universität Hannover, 30167 Hannover, Germany \\ (Received 6 August 2015; revised manuscript received 29 October 2015; published 25 November 2015)
}

\begin{abstract}
We discuss a mechanism for the realization of supersolids in lattices in the absence of intersite interactions that surprisingly works as well at unit filling. This mechanism, that we study for the case of the sawtooth lattice, is based on the existence of frustrated and unfrustrated plaquettes. For sufficiently large interactions and frustration the particles gather preferentially at unfrustrated plaquettes breaking spontaneously translational invariance, resulting in a supersolid. We show that for the sawtooth lattice the supersolid exists for a large region of parameters for densities above half filling. Our results open a feasible path for realizing supersolids in existing ultracold atomic gases in optical lattices without the need for long-range interactions.
\end{abstract}

DOI: 10.1103/PhysRevB.92.195149

PACS number(s): $67.80 . \mathrm{kb}, 67.85 . \mathrm{Hj}$

\section{INTRODUCTION}

Supersolids have attracted large interest since they were proposed [1,2] due to their, apparently counterintuitive, coexistence of both crystalline order and superfluidity. Superfluidity on top of the crystalline order is explained by the creation and delocalization of zero point defects (such as vacancies or interstitials) in a strongly interacting system. The search for the elusive supersolid remains a challenge [3-5]. The claimed evidence of supersolidity in helium [6] was subsequently explained by the shear modulus stiffening of solid ${ }^{4} \mathrm{He}[4,7]$.

Supersolidity may occur as well in lattices due to intersite interactions. Lattice supersolidity has attracted an active theoretical interest as well [8]. Ultracold gases in optical lattices provide an interesting system for the realization of lattice supersolids. However, the requirement of sufficiently large intersite interactions reduces the possible scenarios for supersolidity to gases with long-range interactions, including dipolar gases [8,9] and Rydberg-dressed atomic gases [10,11]. These systems, however, present difficulties due to inelastic collisions in polar molecules [12] and short lifetimes in Rydberg gases. An alternative to polar gases is provided by experiments with condensates in optical cavities, where infinitely long-range interactions between the condensed atoms are induced by two-photon processes. These interactions drive the Dicke phase transition that results in self-organized supersolids [13]. Although these experiments are realized in the absence of optical lattices, the self-organized supersolids spontaneously break a discrete spatial symmetry, hence resembling the case of lattice supersolids.

Long-range interactions are, however, not necessary for the realization of lattice supersolids. Recent studies on frustrated lattices with flat bands, such as kagome lattices [14] and Creutz ladders [15,16], have discussed the possibility of observing supersolids without intersite interactions. In the case of the Creutz ladder, supersolidity at incommensurate densities results from effective next-to-nearest neighbor hopping in the vicinity of the two flat band regions and the doping of a valence bond crystal.

In this paper we discuss a mechanism that leads to robust lattice supersolidity in the absence of intersite interactions. The mechanism is based on the existence of frustrated and unfrustrated plaquettes. In this sense, although we study the specific case of a sawtooth lattice, we expect that supersolids may also be realized in other lattice geometries fulfilling this property. Supersolidity follows from the preferential occupation of unfrustrated plaquettes, which results in spontaneously broken translational symmetry. Sawtooth lattices and other frustrated geometries may be realized using lattice shaking [17] and similar laser arrangements as those recently employed for creating variable lattice geometries [18-20]. Hence our results open a feasible path for realizing supersolidity in existing atomic gases without the need of long-range interactions.

The structure of the paper is as follows. In Sec. II we introduce the sawtooth model studied in the paper. Section III discusses the noninteracting regime. Section IV is devoted to the ground state phase diagram, both at unit filling and away from unit filling. In Sec. V we introduce a simple model that allows an intuitive understanding of the supersolid mechanism. Section VI discusses the roton instability responsible for the superfluid-to-supersolid transition. In Sec. VII we comment on the experimental realization of the sawtooth model and on the signatures of the supersolid phase. Finally, we summarize our conclusions in Sec. VIII.

\section{MODEL}

We consider bosons in the sawtooth lattice as shown in Fig. 1(a), which is characterized by a hopping rate $t^{\prime}<0$ along the lower leg and a hopping rate $t>0$ along the rungs. The change in sign of $t^{\prime}$, which may be achieved experimentally by, e.g., lattice shaking [17], introduces geometric frustration, a key ingredient below. The lower and upper legs have different coordination numbers, and hence constitute two distinct sublattices, which we denote as $A$ and $B$. For a sufficiently deep lattice, the system is described by the Bose-Hubbard model (BHM):

$$
\begin{aligned}
\mathcal{H}= & -t \sum_{i}\left(a_{i}^{\dagger} b_{i}+b_{i}^{\dagger} a_{i+1}+\text { H.c. }\right) \\
& +t^{\prime} \sum_{i}\left(a_{i}^{\dagger} a_{i+1}+\text { H.c. }\right)+\frac{U}{2} \sum_{\nu \in\{A, B\}, i} n_{i}^{v}\left(n_{i}^{v}-1\right),
\end{aligned}
$$

where $a_{i}^{\dagger}\left(b_{i}^{\dagger}\right)$ and $a_{i}\left(b_{i}\right)$ are creation and annihilation operators for bosons at site $i$ of leg $A(B)$, and $n_{i}^{A}=a_{i}^{\dagger} a_{i}\left(n_{i}^{B}=b_{i}^{\dagger} b_{i}\right)$ is the number operator at site $i$ of leg $A(B)$. Contactlike interactions lead to the on-site interaction term, characterized 


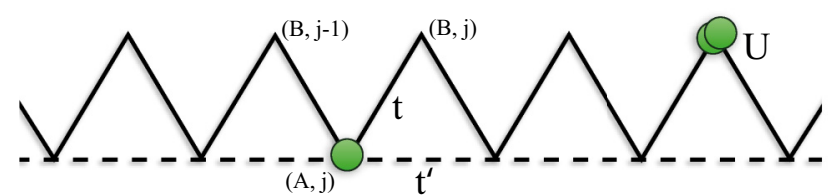

FIG. 1. (Color online) Sawtooth lattice discussed in the paper. The V-shaped plaquettes are unfrustrated, whereas the $\triangle$-shaped plaquettes are frustrated, since we assume $t>0$ and $t^{\prime}<0$. The presence of frustrated and unfrustrated plaquettes is crucial for the supersolid mechanism discussed in the paper.

by the coupling constant $U$. Henceforth we consider all the physical quantities in units of $t=1$ which makes them dimensionless.

\section{NONINTERACTING REGIME}

At first, it is convenient to study the noninteracting regime $(U=0)$. In that case model (1) may be diagonalized in momentum $(k)$ space (note that due to the broken sublattice symmetry, $k \in[-\pi / 2, \pi / 2]): \hat{H}=\sum_{k}\left[E_{\alpha}(k) \alpha_{k}^{\dagger} \alpha_{k}+\right.$ $\left.E_{\beta}(k) \beta_{k}^{\dagger} \beta_{k}\right]$, where the first $(\alpha)$ and second $(\beta)$ energy bands are characterized by the dispersion $E_{\alpha, \beta}(k)=-t^{\prime} \cos 2 k \mp$ $2 t\left[\cos ^{2} k+\left(t^{\prime} / 2 t\right)^{2} \cos ^{2} 2 k\right]^{1 / 2}$ and the bosonic operators $\alpha_{k}=\cos \theta_{k} a_{k}+\sin \theta_{k} b_{k}$ and $\beta_{k}=-\sin \theta_{k} a_{k}+\cos \theta_{k} b_{k}$, with $\tan 2 \theta_{k}=\left(2 t / t^{\prime}\right) \cos k / \cos 2 k$. The lowest band becomes flat at $\left|t^{\prime}\right|=t / \sqrt{2}$ as shown in Fig. 2. When $\left|t^{\prime}\right|<t / \sqrt{2}$ there is a single minimum in the lowest band, whereas two minima occur when $\left|t^{\prime}\right|>t / \sqrt{2}$.

As recently shown [14], for the interacting case the flat band results in a solid phase at lattice filling $\rho=1 / 4$, but the solid order breaks upon doping due to the proliferation of domain walls. In addition, at $\left|t^{\prime}\right|=t / \sqrt{2}$ the minimum of $E_{\alpha}(k)$ changes from $k=0$ to $k=\pi / 2$. As a result, for noninteracting bosons a transition occurs from a superfluid phase at $k=0\left(\mathrm{SF}_{0}\right)$ to a superfluid phase at $k=\pi / 2\left(\mathrm{SF}_{\pi / 2}\right)$. Since $\alpha_{\pi / 2}=a_{\pi / 2}$, in the $\mathrm{SF}_{\pi / 2}$ the $B$ leg is depopulated.

\section{GROUND-STATE PHASE DIAGRAM}

The ground-state phase diagram is obtained by means of numerical density matrix renormalization group (DMRG)

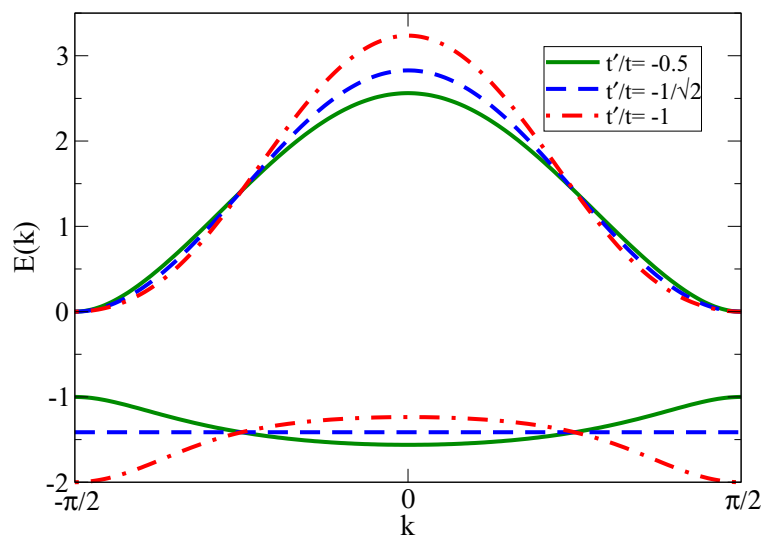

FIG. 2. (Color online) Single particle dispersion for Model (1) with $U=0$ for different values of $\left|t^{\prime}\right| / t$.

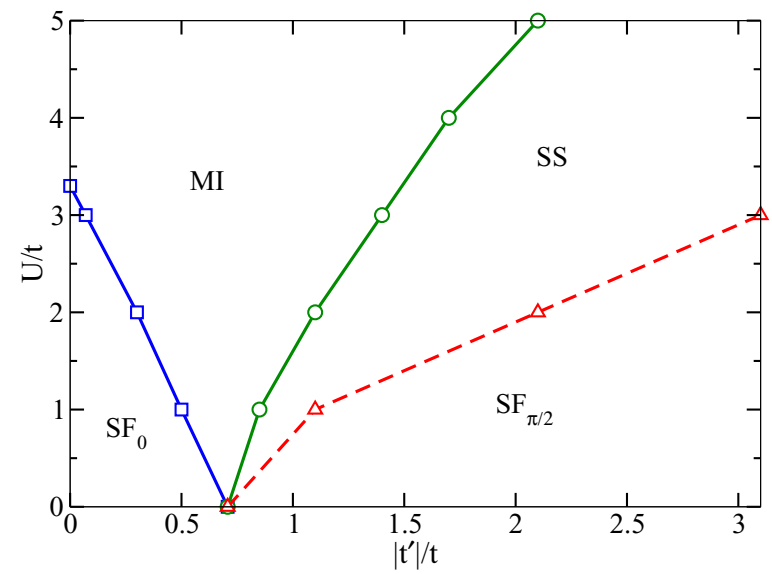

FIG. 3. (Color online) Phase diagram of model (1) for $\rho=1$ as a function of $\left|t^{\prime}\right| / t$ and $U / t$ (see text).

calculations [21-23]. For our calculation we consider up to $L=160$ sites, with maximally four particles per site, retaining up to 500 states in the density matrix. In order to obtain the signature of possible quantum phase transitions in the system, we compute the corresponding order parameters. Particularly relevant is the density structure factor

$$
S(k)=\frac{1}{L^{2}} \sum_{i, j} e^{i k(i-j)}\left\langle n_{i} n_{j}\right\rangle,
$$

where $\left\langle n_{i} n_{j}\right\rangle$ is the density-density correlation between sites $i$ and $j$.

In order to quantify the critical point for the $\mathrm{SF}_{0}$ to the Mott-insulator (MI) transition discussed below, we calculate the Luttinger parameter $K$, which is the power-law exponent of the decay of the single particle correlation function $\Gamma_{r}=$ $\left\langle a_{i}^{\dagger} a_{j}\right\rangle$. However, in order to obtain the value of $K$ we evaluate the structure factor $S(k)$ in the long wavelength limit $(k \rightarrow 0)$, since in this limit [24]

$$
S(k) \propto K|k| / 2 \pi .
$$

We also compute the single particle gap to characterize the gapped MI phase as

$$
E_{G}=\mu^{+}-\mu^{-},
$$

where $\mu^{+}\left(\mu^{-}\right)$is the chemical potential for adding (removing) one particle. Finally, the momentum distribution function

$$
N(k)=\frac{1}{L} \sum_{i, j} e^{i k(i-j)}\left\langle a_{i}^{\dagger} a_{j}\right\rangle
$$

is particularly interesting as a possible experimental signature of the supersolid phase discussed below. In the following subsections we discuss the ground-state phase diagram at unit filling and away from unit filling.

\section{A. Unit filling}

Figure 3 shows the ground-state phase diagram of model (1) at unit filling, $\rho=1$. As discussed above, in the noninteracting limit the system exhibits two gapless superfluid phases, $\mathrm{SF}_{0}$ and $\mathrm{SF}_{\pi / 2}$. In addition to these phases, the system opens, 


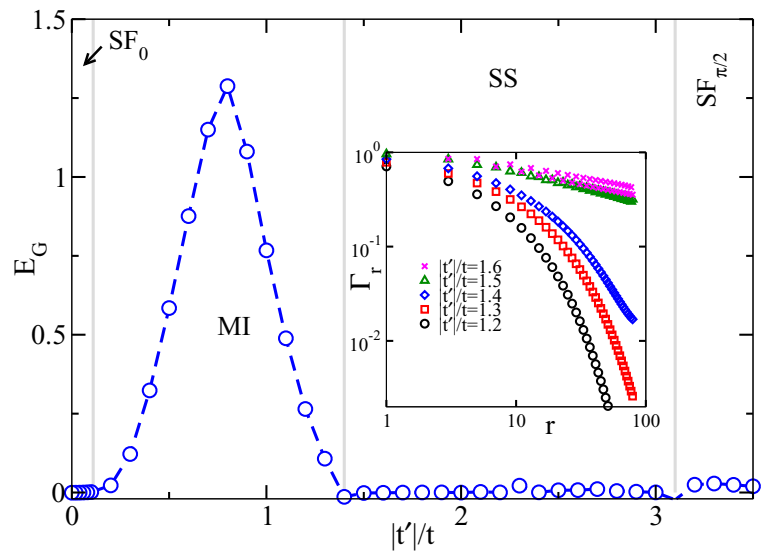

FIG. 4. (Color online) Extrapolated gap $E_{G}$ as a function of $\left|t^{\prime}\right| / t$. The inset shows the exponential to power-law decay of the single particle correlation function $\Gamma_{r}$ at the MI-SS boundary. Only odd $r$ is shown to avoid oscillations.

as expected, a MI phase at small $U$, which at the flat-band point is maintained all the way down to vanishing $U$. The second additional phase is, in contrast, rather unexpected. As we discuss below, this phase is a supersolid (SS), which rather surprisingly occurs at $\rho=1$ and in the absence of intersite interactions.

In the following we discuss in detail the different phases for the case $U / t=3$. In Fig. 4 we plot the thermodynamic limit values of the single-particle gap [Eq. (4)]. The MI region is characterized by a finite gap, whereas $E_{G} \rightarrow 0$ in all other regions. The smooth gap opening at small $\left|t^{\prime}\right| / t$ marks the Berezinskii-Kosterlitz-Thouless (BKT) $\mathrm{SF}_{0}$-to-MI transition, characterized by a Luttinger parameter $K=2$ [24]. By performing a finite size scaling of $K$ computed using Eq. (3), we obtain that the transition for $\mathrm{SF}_{0}$ to $\mathrm{MI}$ phase occurs at $\left|t^{\prime}\right| / t \simeq 0.1$ [see Fig. 5(a)]. The BKT transition is further confirmed by the scaling of the momentum distribution, $N(k=0) \propto L^{1-\frac{1}{2 K}}$ [25-27]. In Fig. 5(b) we show that the values of $N(k=0) L^{-3 / 4}$ for different lengths $(L=80,120$, 160) intersect at the transition point $\left|t^{\prime}\right| / t \simeq 0.1$.

In contrast, the sudden vanishing of $E_{G}$ for larger $\left|t^{\prime}\right| / t$ (at $\left|t^{\prime}\right| / t=1.4$ in Fig. 4) does not match with a BKT transition. (a)

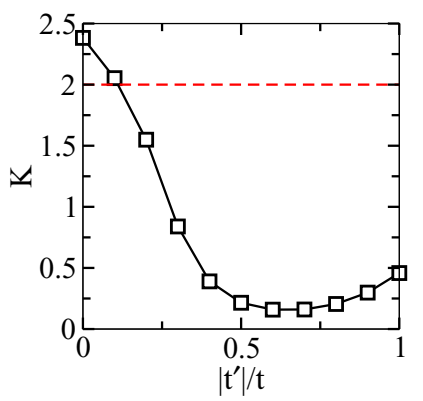

(b)

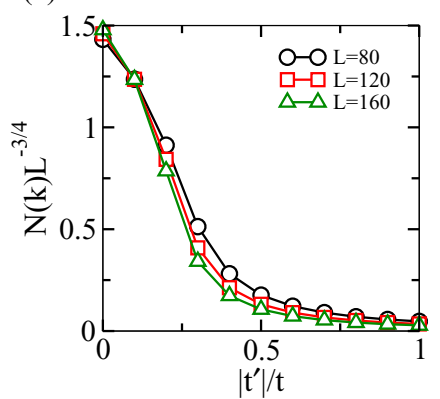

FIG. 5. (Color online) (a) Extrapolated values of $K$ (black squares) with respect to $\left|t^{\prime}\right| / t$ for $U / t=3$. The $K=2$ line (red dashed) is drawn to find the critical point for the $\mathrm{SF}_{0}-\mathrm{MI}$ transition. (b) Scaled momentum distribution $N(k) L^{-3 / 4}$ for different lengths as a function of $\left|t^{\prime}\right| / t$ for $U / t=3$.

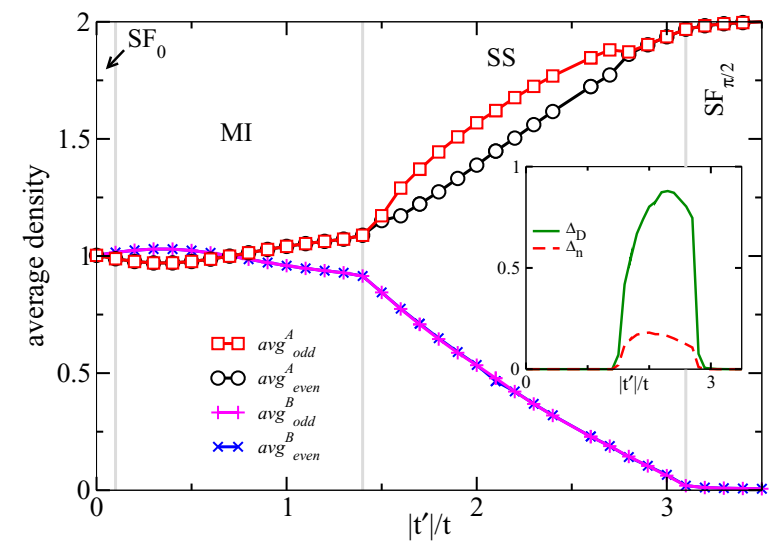

FIG. 6. (Color online) Number fluctuation between odd and even sites of the $A$ and $B$ legs; the inset shows $\Delta_{n}$ and $\Delta_{D}$.

The gapped to gapless transition is, however, confirmed by the single-particle correlation function $\Gamma_{r}=\left\langle a_{i}^{\dagger} a_{i+r}\right\rangle$, which decays exponentially in the MI and algebraically for $\left|t^{\prime}\right| / t>$ 1.4 (inset of Fig. 4). This transition is likely to be a weak first order transition which could not be confirmed in our numerics.

The superfluid region opening at the large $\left|t^{\prime}\right| / t$ side of the MI turns out to be a SS phase. Figure 6 shows the average density $a v g_{\text {odd,even }}^{A}\left(a v g_{\text {odd,even }}^{B}\right)$ at even and odd sites in the $A$ $(B)$ leg. The difference of densities at both legs is not surprising due to the asymmetry between the legs. More interesting is the behavior within a given leg. Whereas $a v g_{\text {odd }}^{B}=a v g_{\text {even }}^{B}$ for all $\left|t^{\prime}\right| / t, \operatorname{avg}_{\text {odd }}^{A} \neq \operatorname{avg}_{\text {even }}^{A}$ in the SS region $\left(1.4<\left|t^{\prime}\right| / t<\right.$ 3.1 in Fig. 6) indicating a clear density modulation in this region. The spontaneously broken translational symmetry along the $A$ leg is characterized by the amplitude $\Delta_{n}=$ $a v g_{\text {odd }}^{A}-a v g_{\text {even }}^{A}$ of the odd-even modulation (inset of Fig. 6). The broken symmetry translates as well in a difference of bondkinetic energy $\Delta_{D}=a v g_{\text {odd }}^{D}-a v g_{\text {even }}^{D}$, where $a v g_{\text {odd,even }}^{D}=$ $\frac{1}{L} \sum_{i \in A_{\text {odd even }}}\left(b_{i-1}^{\dagger} a_{i}+a_{i}^{\dagger} b_{i+1}+\right.$ H.c. $)$, which is finite in the $\mathrm{SS}$ region (inset of Fig. 6). The finite $\Delta_{D}$ relates to the $V$-type dimerization discussed below. The end of the SS region and the onset of the $\mathrm{SF}_{\pi / 2}$ phase is marked by the vanishing of both $\Delta_{n}$ and $\Delta_{D}$ and the depopulation of the $B$ leg.

The SS phase can be further confirmed by a finite peak in $S(k)$ at nonzero wave vector $k$. However, we note that the sawtooth lattice breaks a trivial translational symmetry due to which $S(k)$ shows a peak at $k= \pm \pi$. Therefore, the presence of the SS phase in the system is revealed by a finite peak in the structure factor at $k= \pm \pi / 2$, confirmed by extrapolation to the thermodynamic limit. In Fig. 7 (top) we show, for $U / t=$ 3 , the extrapolated value of $S(k=\pi / 2)$, which is finite in the region $1.4<\left|t^{\prime}\right| / t<3.1$, i.e., the $\mathrm{SS}$ phase. The extrapolation of $S(k=\pi / 2)$ is shown in detail in Fig. 7 (bottom).

\section{B. Away from unit filling}

At this point we consider incommensurate filling. This discussion is particularly relevant, since the presence of an overall harmonic trap, typical of experiments on atoms in optical lattices, results in an inhomogeneous density distribution. In Fig. 8 we depict our results for $U / t=2$. In addition to the gapped density-wave phase at $\rho=0.25$ at the flat-band point 

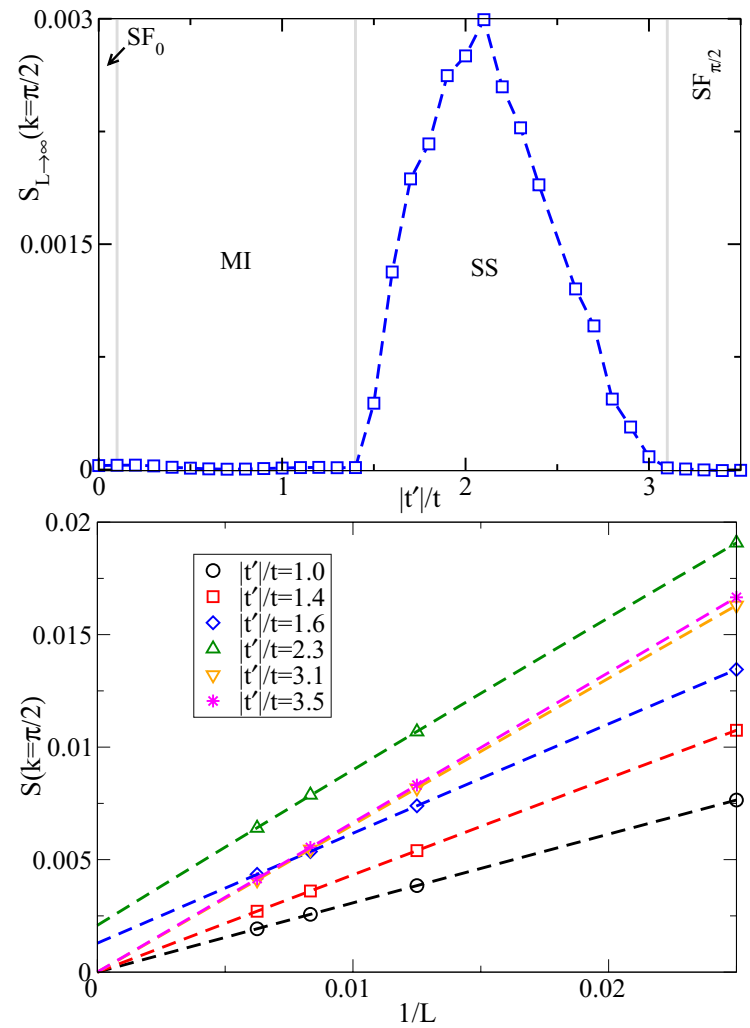

FIG. 7. (Color online) (Top) Scaled structure factor $S(k=\pi / 2)$ with respect to $\left|t^{\prime}\right| / t$ for $U / t=3.0$. (Bottom) Finite-size scaling of $S(k=\pi / 2)$ for $U / t=3.0$ and different $\left|t^{\prime}\right| / t$ values.

[14], we obtain gapped phases at $\rho=n / 4$ for all integer $n \geqslant 1$ (black lines and squares in Fig. 8). The gapped phases at these densities are expected to occur at the large interaction limit. The phases at $\rho=(2 n+1) / 4$ with $n>1$ are similar to the phase at $\rho=1 / 4$, being comparatively narrow due to small

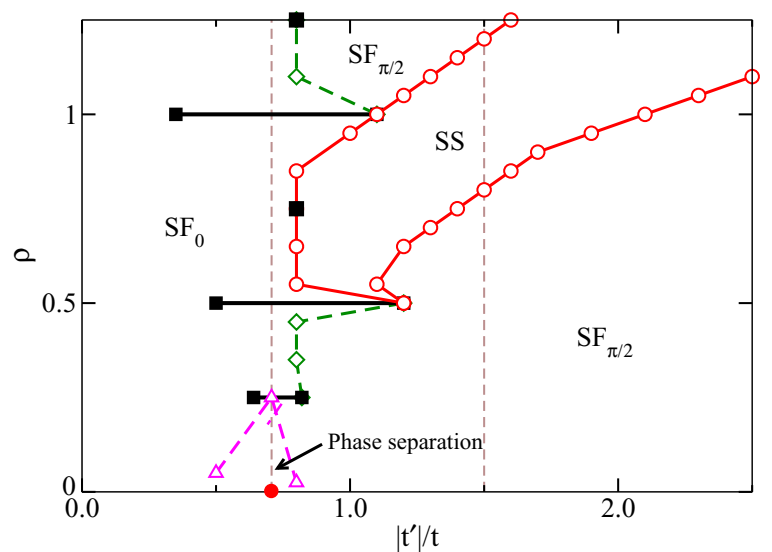

FIG. 8. (Color online) Phase diagram of the sawtooth chain as a function of $\rho$ and $\left|t^{\prime}\right| / t$ for $U / t=2.0$. Black lines with squares show the gapped regions. The SS phase is bounded by the red circles. The $\mathrm{SF}_{\pi / 2}$ phase occupies a large portion of the phase diagram below the SS region and for $\left|t^{\prime}\right| / t>1 / \sqrt{2}$, also around $\rho=1$. The region bounded by magenta triangles shows a macroscopic jump in the density. The brown dashed lines are two representative cuts at $\left|t^{\prime}\right| / t=$ $1 / \sqrt{2}$ and 1.5 (see text). The red dot corresponds to the flat-band point where $\left|t^{\prime}\right| / t=1 / \sqrt{2}$.

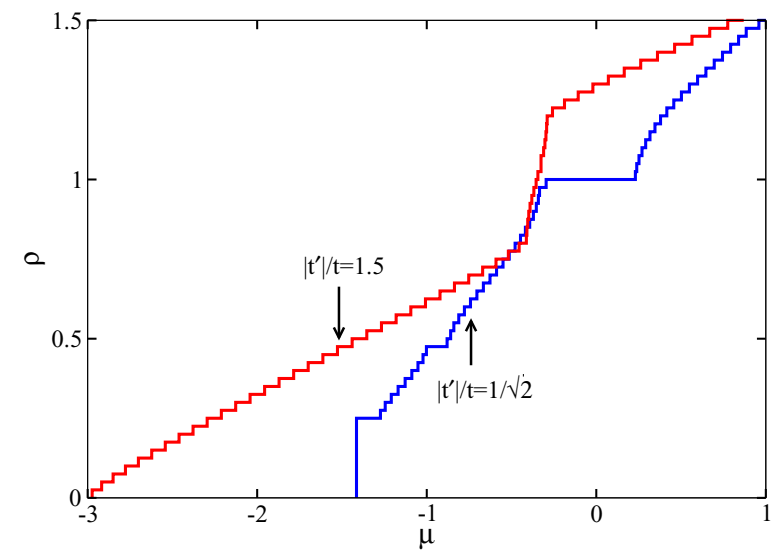

FIG. 9. (Color online) $\rho(\mu)$ for $L=40, U / t=2$, and $\left|t^{\prime}\right| / t=$ $1 / \sqrt{2}$ (blue curve) and 1.5 (red curve).

interaction. On the contrary, the gapped phase at $\rho=1 / 2$ is a trivial insulator due to a filled band. Even more relevant is the robustness of the SS phase, which extends over a large parameter region, for densities $\rho>0.5$.

We illustrate the behavior of the system for the case of two representative values, $\left|t^{\prime}\right|=1 / \sqrt{2}$ and 1.5 (brown dashed lines in Fig. 8). In Fig. 9, we plot $\rho$ as a function of the chemical potential $\mu$ for $\left|t^{\prime}\right| / t=1 / \sqrt{2}$. Due to band flatness at that point, at a critical $\mu$ there exists a macroscopic jump in the density up to $\rho=0.25$. This macroscopic jump exists for a small region around $\left|t^{\prime}\right| / t=1 / \sqrt{2}$. This region is marked in the phase diagram by magenta triangles. At $\rho=0.25$ a gap appears marked by a plateau in $\rho(\mu)$. Then, the system becomes a gapless and compressible superfluid. Further increase in $\rho$ induces additional gaps as discussed above. In between the gapped plateaus the system is a superfluid.

The dependence of $\rho(\mu)$ is very different for $\left|t^{\prime}\right| / t=1.5$, since for that value the band is not flat. As a result the system starts at $\rho \rightarrow 0$ in the $\mathrm{SF}_{\pi / 2}$ phase. In Fig. 9 we observe that the curve $\rho(\mu)$ is continuous with two kinks at $\rho \simeq 0.8$ and 1.2. These kinks correspond to the transition to the SS phase. The abrupt growth of $\rho$ in the SS phase shows that the SS phase is highly compressible.

Finally, Fig. 10 shows $S(k=\pi / 2)$ for $\left|t^{\prime}\right| / t=1 / \sqrt{2}$ and 1.5. For $\left|t^{\prime}\right| / t=1.5, S(k=\pi / 2)$ increases in the SS phase, $0.8<\rho<1.2$. In contrast, for $\left|t^{\prime}\right| / t=1 / \sqrt{2}, S(k=\pi / 2)$ remains small except at three peaks at $\rho=0.25,0.75$, and 1.25. The two small peaks at $\rho=0.75$ and 1.25 are due to the presence of two small density-wave phases shown as black squares in Fig. 8. The sharp peak at $\rho=0.25$ corresponds to the density-wave phase reported in Ref. [14].

\section{WEAK COUPLING LIMIT}

An intuitive insight on the emergence of the SS is obtained from the classical limit of model (1). We assume each site to be in a coherent state with a well defined density and phase, 0 or $\pi$, corresponding to the two possible minima of $E_{\alpha}(k)$. We consider a simplified model in which $b_{j}=\eta, a_{2 j}=\xi$, and $a_{2 j+1}=\chi$, such that we allow for both a possible density imbalance between the $A$ and $B$ legs and for an even-odd 


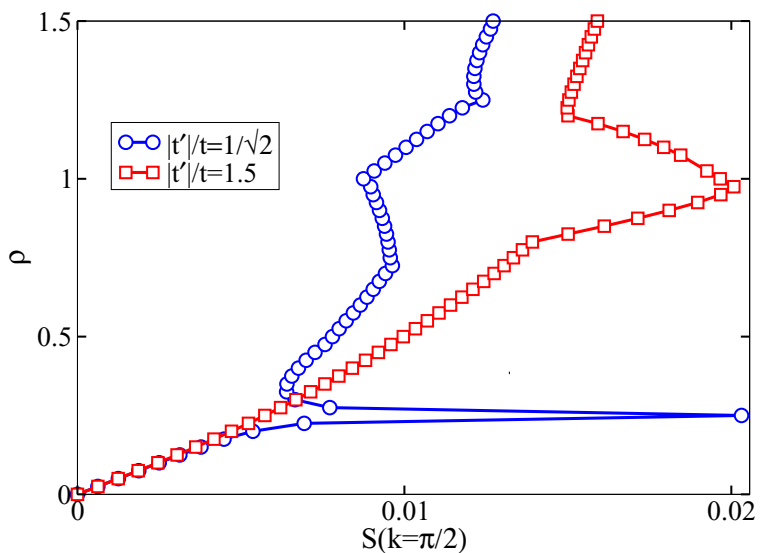

FIG. 10. (Color online) Structure factor $S(k=\pi / 2)$ for different fillings $\rho$ for $L=40, U / t=2$, and $\left|t^{\prime}\right| / t=1 / \sqrt{2}$ (blue circles) and 1.5 (red squares).

asymmetry in the $A$ leg. We may hence minimize the energy, which without loss of generality may be calculated for a foursite unit cell:

$$
\begin{aligned}
\langle\mathcal{H}\rangle= & -4 t(\xi \chi+\chi \eta)-4 t^{\prime} \xi \eta \\
& +U\left(\xi^{4}+2 \chi^{4}+\eta^{4}\right)-\mu\left(\xi^{2}+2 \chi^{2}+\eta^{2}\right)
\end{aligned}
$$

Within this approach the phase diagram splits into three regions [Fig. 11(d)]. For $\left|t^{\prime}\right| / t<1 / \sqrt{2}$ the three coefficients have the same sign, and the particles occupy both $A$ and $B$ sites corresponding to the $\mathrm{SF}_{0}$ phase [Fig. 11(a)]. For small $U / t$ and $\left|t^{\prime}\right| / t>1 / \sqrt{2}$, the $B$ sites depopulate $(\eta=$ $0)$, the density is homogeneous in the $A$ sites $(|\chi|=|\xi|)$, and $\operatorname{sign}(\chi) \neq \operatorname{sign}(\xi)$, corresponding to the $\mathrm{SF}_{\pi / 2}$ phase [Fig. 11(b)]. A sufficiently strong repulsive interaction $U / t>$ $(U / t)_{c}$ redistributes population to the $B$ sites. However, how the particles redistribute in the $A$ leg is crucially determined by the existence of frustrated and unfrustrated plaquettes in the sawtooth lattice. In order to minimize kinetic energy particles favor the unfrustrated V-shaped plaquettes of the sawtooth

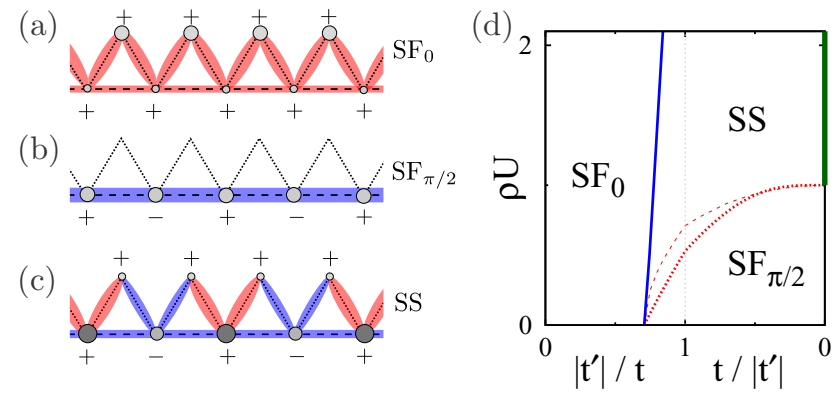

FIG. 11. (Color online) Sketch of the $\mathrm{SF}_{0}$ (a), $\mathrm{SF}_{\pi / 2}$ (b), and SS (c). The circle size is proportional to the local density, whereas \pm denote the sign. (d) Phase diagram in the weak-coupling limit as function $U \rho$ and $\left|t^{\prime}\right| / t$ for a fixed (but arbitrary) filling. The $\mathrm{SF}_{0}$ to the SS phase is shown as a solid line. The dotted and dashed lines depict, respectively, the $\mathrm{SF}_{\pi} / 2-\mathrm{SS}$ transition line obtained from the variational approach (6) and the roton instability. At $t=0 \mathrm{SS}$-order vanishes (thick solid line) resulting in a highly degenerate ground state, since particles may occupy the uncoupled $B$ sites in an arbitrary configuration. (a)

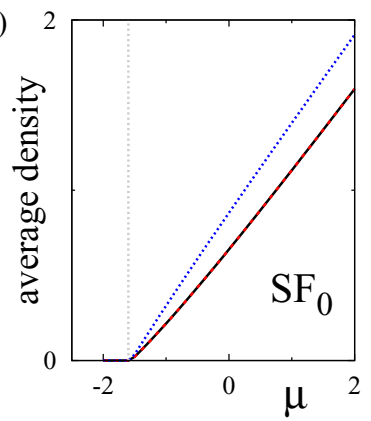

(b)

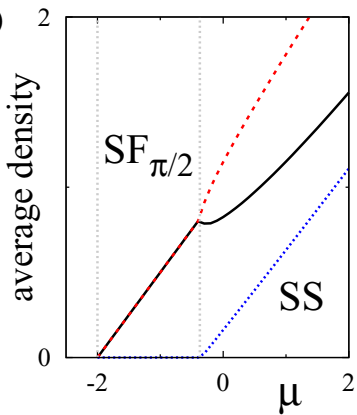

FIG. 12. (Color online) Densities of the classical model for $\rho U=2$ and (a) $t=1$ and $t^{\prime}=-0.5$ and (b) $t=0.8$ and $t^{\prime}=-1$ as a function of the chemical potential $\mu$. The dotted (blue) line depicts the average density of the $B$ sites; the solid (black) and dashed (red) lines show densities of odd and even $A$ sites.

forming V-shaped dimers. As a result particles break the translational symmetry spontaneously, preferably occupying every second $\mathrm{V}$ plaquette [Fig. 11(c)], which leads to a density modulation in the $A$ sites that characterizes, as mentioned above, the SS phase. Note that this simple picture also predicts a finite $\Delta_{D}$ in the SS region, as observed in the numerics.

As discussed above, already the classical or weak-coupling limit gives intuitive insight into the formation of the SS phase. We provide in this section some additional details. In Fig. 12 we show typical densities as a function of $\mu$, obtained using model (6). While in the $\mathrm{SF}_{0}$ and $\mathrm{SF}_{\pi / 2}$ phases the $A$ sites do not exhibit any density modulation, when entering the SS phase the $a v g_{\text {even }}^{A}$ and $a v g_{\text {odd }}^{A}$ curves separate from each other. The amplitude of the modulation on the $A$ sites in the limit $\mu \gg$ $t,\left|t^{\prime}\right|, U$ is given by $\xi-\chi=\frac{2 t}{\sqrt{U \mu}}$, suppressed with increasing repulsion $U$. Within this approach the population of the $B$ sites in the $\mathrm{SF}_{\pi / 2}$ phase is strictly zero. We determine the $\mathrm{SF}_{0}$ to $\mathrm{SS}$ transition as first-order, whereas the $\mathrm{SF}_{\pi / 2}$ to $\mathrm{SS}$ transition is a second-order phase transition exhibiting a discontinuity in second derivative of the ground-state energy.

\section{ROTON INSTABILITY}

Insight on the nature of the $\mathrm{SF}_{\pi / 2}$-SS transition is obtained in the limit in which $U \ll E_{\beta}(k)-E_{\alpha}(k)$ for all $k$. In that case we may project model (1) onto the lowest energy band:

$$
H \simeq \sum_{k} E_{\alpha}(k) \alpha_{k}^{\dagger} \alpha_{k}+\frac{U}{2} \sum_{q, k, k^{\prime}} f_{k, k^{\prime}}^{k+q, k^{\prime}-q} \alpha_{k+q}^{\dagger} \alpha_{k^{\prime}-q}^{\dagger} \alpha_{k^{\prime}} \alpha_{k}
$$

with $f_{k_{1}, k_{2}}^{k_{3}, k_{4}}=\prod_{l=1}^{4} \cos \left(\theta_{k_{l}}\right)+\prod_{l=1}^{4} \sin \left(\theta_{k_{l}}\right)$. Note that, although the on-site interactions are contactlike, the effective interactions are momentum dependent.

Starting from Eq. (7) and after expanding the interaction part up to second order terms assuming condensation at $q=\pi / 2$, i.e., $\alpha_{q} \simeq \sqrt{N}+\tilde{\alpha}_{q}$, the total Hamiltonian may be written up to constants as $\mathcal{H} \simeq \sum_{k>0} A(k)\left(\tilde{\alpha}_{k}^{\dagger} \tilde{\alpha}_{k}+\tilde{\alpha}_{-k}^{\dagger} \tilde{\alpha}_{-k}\right)+$ $B(k)\left(\tilde{\alpha}_{k}^{\dagger} \tilde{\alpha}_{-k}^{\dagger}+\tilde{\alpha}_{k} \tilde{\alpha}_{-k}\right)$ with

$$
\begin{aligned}
A(k)= & E_{\alpha}(k)+\rho U\left[\cos 2 \theta_{q}\left(\cos 2 \theta_{k}-\cos 2 \theta_{q}\right)\right. \\
& \left.+\left(1+\cos 2 \theta_{q} \cos 2 \theta_{k}\right)\right] \\
B(k)= & \rho U\left(1+\cos 2 \theta_{q} \cos 2 \theta_{k}\right) .
\end{aligned}
$$




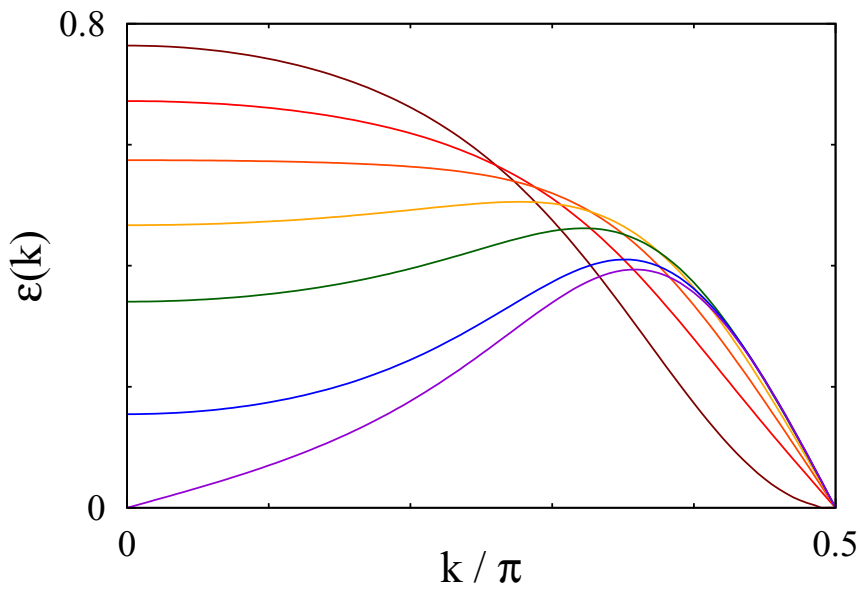

FIG. 13. (Color online) Bogoliubov spectrum $\epsilon(k)$ for $\left|t^{\prime}\right| / t=1$ and (from top to bottom) $\rho U=0,0.1,0.2,0.3,0.4,0.5,0.5278$. At $k=0$ a rotonlike minimum develops, which touches zero at $\rho U \simeq$ 0.5278 .

This Hamiltonian may be readily diagonalized using a Bogoliubov transformation $\beta_{k}=\cosh \gamma_{k} \tilde{\alpha}_{k}-\sinh \gamma_{k} \tilde{\alpha}_{-k}^{\dagger}$ yielding the Bogoliubov spectrum of excitations, $\epsilon(k)^{2}=$ $\left(\widetilde{E_{A}}(k)+2 U \rho\left(1+\cos 2 \theta_{k}\right)\right) \widetilde{E_{A}}(k)$ with $\widetilde{E_{A}}(k)=E_{A}(k)-$ $2 t^{\prime}+U \rho\left(\cos 2 \theta_{k}-1\right)$.

In Fig. 13 we depict the corresponding energy spectrum emerging for finite $\rho U$. As usual the spectrum exhibits a linear (phononlike) dispersion for $k$ close to $\pi / 2$. Interestingly, for finite $\rho U$ it acquires a local minimum at $k=0$ that resembles the roton dispersion minimum of superfluid He [28], and that occurs, as for dipolar condensates [29], due to the momentum dependence of the interactions. For a critical value of $\rho U$ the rotonlike minimum reaches zero energy, becoming unstable, marking the transition to the SS. As shown in Fig. 11(d) the critical $\rho U$ for roton instability agrees well with the $\mathrm{SF}_{\pi / 2}-\mathrm{SS}$ transition line obtained by the classical model. Hence, we can conclude that the $\mathrm{SF}_{\pi / 2}$ is destabilized through roton instability that leads to the SS phase.

\section{EXPERIMENTAL SIGNATURE}

In the section we briefly discuss the possible signatures of the SS phase in optical lattice experiments using ultracold atoms. The sawtooth lattice can be created by suitably using a

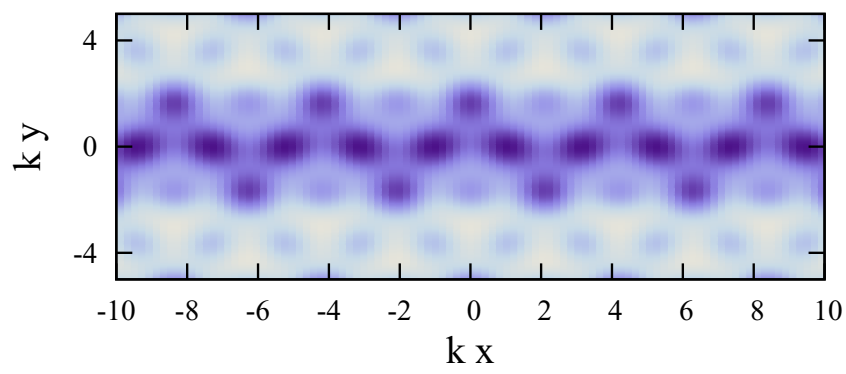

FIG. 14. (Color online) Sawtooth lattice formed by an incoherent superposition between a kagome lattice [20] and an additional lattice $V(\vec{r})=\sin ^{2}\left(\frac{\sqrt{3}}{4} k y\right)+\frac{1}{4} \sin ^{2}\left(\frac{\sqrt{3}}{2} k y\right)$ with $k$ the laser wave number. Darker regions mean lower potential.

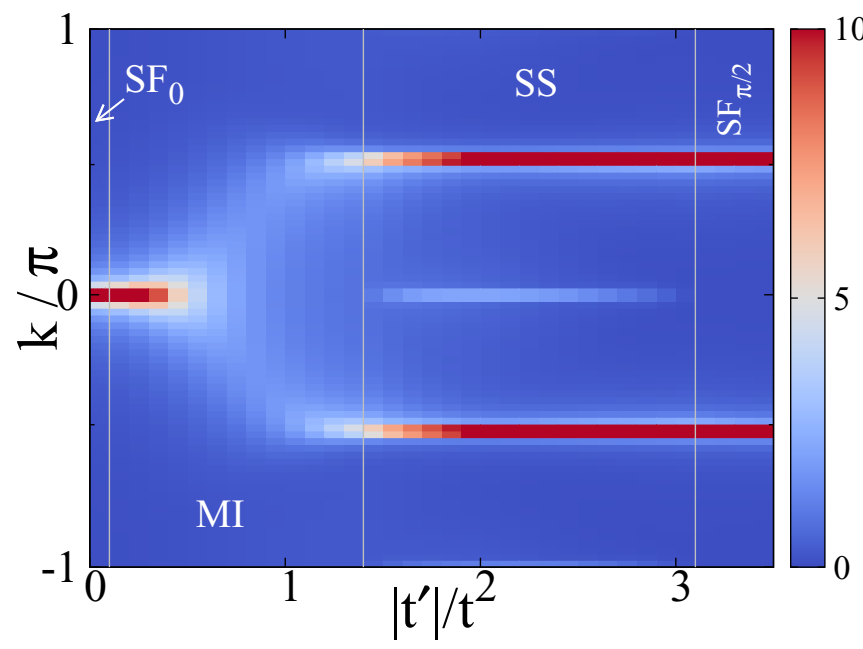

FIG. 15. (Color online) Momentum distribution $N(k)$ in different regions of the phase diagram shown in Fig. 3 for $U=3$.

superlattice potential on top of the kagome lattice which has been created recently [20] (see Fig. 14).

In order to obtain the signature of the SS phase we compute the momentum distribution $N(k)$ using Eq. (5). As expected, in $\mathrm{SF}_{0}\left(\mathrm{SF}_{\pi / 2}\right) N(k)$ has a peak at $k=0(k=\pi / 2)$. However, an intriguing feature appears in the SS phase which shows, interestingly, peaks at $k=0$ and $k=\pi / 2$ as shown in Fig. 15 . We also plot the peak strengths as a function of $\left|t^{\prime}\right| / t$ at $k=0$ and $k=\pi / 2$ in Fig. 16, which shows the transition from $\mathrm{SF}_{0^{-}}$ MI-SS-SF ${ }_{\pi / 2}$ for $U=3$ and $\rho=1$. Hence, the appearance of the SS phase may be directly monitored in time-of-flight experiments from the multipeaked momentum distribution.

\section{CONCLUSIONS}

We have discussed a mechanism for the formation of lattice supersolids for the particular case of a sawtooth lattice. The mechanism is based on the selective population of unfrustrated plaquettes in the presence of frustrated and unfrustrated plaquettes, and hence we expect supersolids in other lattices fulfilling that property. We have shown that the supersolid exists for a broad range of lattice fillings, including

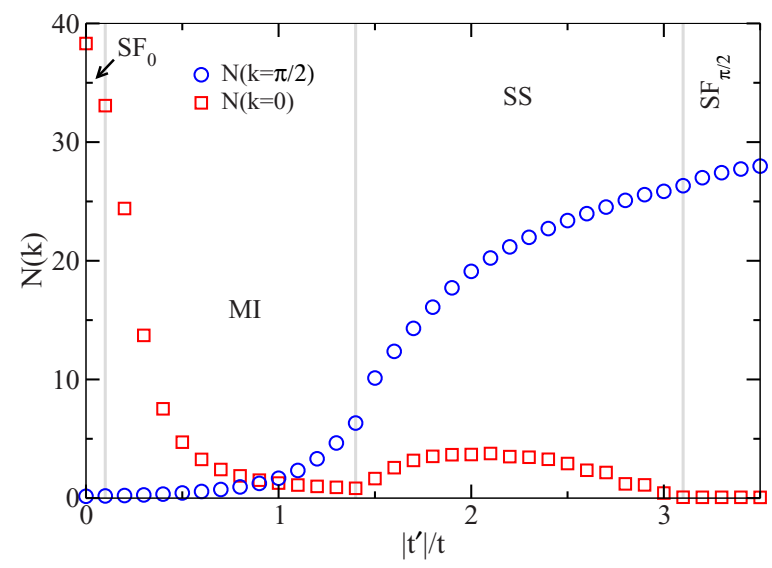

FIG. 16. (Color online) $N(0)$ (red squares) and $N(\pi / 2)$ (blue circles) in different regions of the phase diagram shown in Fig. 3 at $U=3$. 
various commensurate fillings, in particular unit filling. Interestingly the supersolid may be revealed not only by in situ measurements but by monitoring the momentum distribution in time-of-flight measurements. Since frustrated lattices, in particular sawtooth, may be realized using state of the art techniques, our results hence open a feasible path for realizing supersolids in existing experiments with ultracold atoms in optical lattices, without the need for long-range interactions.

\section{ACKNOWLEDGMENTS}

We thank T. Vekua for enlightening discussions. We acknowledge support from the Center for Quantum Engineering and Space Time Research and the Deutsche Forschungsgemeinschaft (Research Training Group 1729). Simulations were performed on the cluster system of the Leibniz Universität Hannover.
[1] A. F. Andreev and I. M. Lifshitz, Sov. Phys. JETP 29, 1107 (1969).

[2] G. V. Chester, Phys. Rev. A 2, 256 (1970).

[3] N. Prokof'ev and B. Svistunov, Phys. Rev. Lett. 94, 155302 (2005).

[4] M. Boninsegni and N. Prokof'ev, Rev. Mod. Phys. 84, 759 (2012).

[5] S. Balibar, Nature (London) 464, 176 (2010).

[6] E. Kim and M. H. W. Chan, Nature (London) 427, 225 (2004); Science 305, 1941 (2004).

[7] A. B. Kuklov, N. V. Prokof'ev, and B. V. Svistunov, Physics 4, 109 (2011).

[8] See M. A. Baranov, M. Dalmonte, G. Pupillo, and P. Zoller, Chem. Rev. 112, 5012 (2012), and references therein.

[9] T. Lahaye, C. Menotti, L. Santos, M. Lewenstein, and T. Pfau, Rep. Prog. Phys. 72, 126401 (2009).

[10] T. F. Gallagher and P. Pillet, Advances In Atomic, Molecular, and Optical Physics, Vol. 56, edited by E. Arimondo, P. R. Berman, and C. C. Lin (Academic Press, London, 2008), p. 161.

[11] F. Cinti, T. Macrì, W. Lechner, G. Pupillo, and T. Pohl, Nat. Commun. 5, 3235 (2014).

[12] S. Ospelkaus, K.-K. Ni, D. Wang, M. H. G. de Miranda, B. Neyenhuis, G. Quéméner, P. S. Julienne, J. L. Bohn, D. S. Jin, and J. Ye, Science 327, 853 (2010).

[13] K. Baumann, C. Guerlin, F. Brennecke, and T. Esslinger, Nature (London) 464, 1301 (2010).

[14] S. D. Huber and E. Altman, Phys. Rev. B 82, 184502 (2010).

[15] S. Takayoshi, H. Katsura, N. Watanabe, and H. Aoki, Phys. Rev. A 88, 063613 (2013).

[16] M. Tovmasyan, E. P. L. van Nieuwenburg, and S. D. Huber, Phys. Rev. B 88, 220510(R) (2013).
[17] J. Struck, C. Ölschlger, R. Le Targat, P. Soltan-Panahi, A. Eckardt, M. Lewenstein, P. Windpassinger, and K. Sengstock, Science 333, 996 (2011).

[18] G. Wirth, M. Ölschläger, and A. Hemmerich, Nat. Phys. 7, 147 (2011).

[19] L. Tarruell, D. Greif, T. Uehlinger, G. Jotzu, and T. Esslinger, Nature (London) 483, 302 (2012).

[20] G.-B. Jo, J. Guzman, C. K. Thomas, P. Hosur, A. Vishwanath, and D. M. Stamper-Kurn, Phys. Rev. Lett. 108, 045305 (2012).

[21] S. R. White, Phys. Rev. Lett. 69, 2863 (1992).

[22] U. Schollwoeck, Rev. Mod. Phys. 77, 259 (2005).

[23] The sawtooth chain considered has an even number of sites so that we have an extra site in the end of the lattice. This assumption enables us to get rid of any possible degenerate dimerized phase which may possibly occur in the large $U$ limit [see O. Derzhko, J. Richter, and M. Maksymenko, Int. J. Mod. Phys. B 29, 1530007 (2015)].

[24] T. Giamarchi, Quantum Physics in One Dimension, International Series of Monographs on Physics, Vol. 121 (Oxford University Press, Oxford, 2004).

[25] A. Dhar, M. Maji, T. Mishra, R. V. Pai, S. Mukerjee, and A. Paramekanti, Phys. Rev. A 85, 041602(R) (2012).

[26] A. Dhar, T. Mishra, M. Maji, R. V. Pai, S. Mukerjee, and A. Paramekanti, Phys. Rev. B 87, 174501 (2013).

[27] S. Greschner, L. Santos, and T. Vekua, Phys. Rev. A 87, 033609 (2013).

[28] L. D. Landau, J. Phys. USSR 11, 91 (1947); Phys. Rev. 75, 884 (1949).

[29] L. Santos, G. V. Shlyapnikov, and M. Lewenstein, Phys. Rev. Lett. 90, 250403 (2003). 